\title{
DGSAT: Dwarf Galaxy Survey with Amateur Telescopes
}

\section{A catalogue of isolated nearby edge-on disk galaxies and the discovery of new low surface brightness systems}

\author{
C. Henkel ${ }^{1,2}$, B. Javanmardi ${ }^{3}$, D. Martínez-Delgado ${ }^{4}$, P. Kroupa ${ }^{5,6}$, and K. Teuwen ${ }^{7}$ \\ 1 Max-Planck-Institut für Radioastronomie, Auf dem Hügel 69, 53121 Bonn, Germany \\ e-mail: chenkel@mpifr-bonn.mpg.de \\ 2 Astronomy Department, Faculty of Science, King Abdulaziz University, PO Box 80203, 21589 Jeddah, Saudi Arabia \\ 3 Argelander Institut für Astronomie, Universität Bonn, Auf dem Hügel 71, 53121 Bonn, Germany \\ 4 Astronomisches Rechen-Institut, Zentrum für Astronomie, Universität Heidelberg, Mönchhofstr. 12-14, 69120 Heidelberg, \\ Germany \\ 5 Helmholtz Institut für Strahlen- und Kernphysik (HISKP), Universität Bonn, Nussallee 14-16, 53121 Bonn, Germany \\ ${ }^{6}$ Charles University, Faculty of Mathematics and Physics, Astronomical Institute, V Holešovičkách 2, 18000 Praha 8 , \\ Czech Republic \\ 7 Remote Observatories Southern Alps, Verclause, France \\ Received 31 January 2017 / Accepted 7 March 2017
}

\begin{abstract}
The connection between the bulge mass or bulge luminosity in disk galaxies and the number, spatial and phase space distribution of associated dwarf galaxies is a discriminator between cosmological simulations related to galaxy formation in cold dark matter and generalised gravity models. Here, a nearby sample of isolated Milky Way-class edge-on galaxies is introduced, to facilitate observational campaigns to detect the associated families of dwarf galaxies at low surface brightness. Three galaxy pairs with at least one of the targets being edge-on are also introduced. Approximately $60 \%$ of the catalogued isolated galaxies contain bulges of different size, while the remaining objects appear to be bulgeless. Deep images of NGC 3669 (small bulge, with NGC 3625 at the edge of the image) and NGC 7814 (prominent bulge), obtained with a $0.4 \mathrm{~m}$ aperture, are also presented, resulting in the discovery of two new dwarf galaxy candidates, NGC 3669-DGSAT-3 and NGC 7814-DGSAT-7. Eleven additional low surface brightness galaxies are identified, previously notified with low quality measurement flags in the Sloan Digital Sky Survey (SDSS). Integrated magnitudes, surface brightnesses, effective radii, Sersic indices, axis ratios, and projected distances to their putative major hosts are displayed. At least one of the galaxies, NGC 3625-DGSAT-4, belongs with a surface brightness of $\mu_{\mathrm{r}} \approx 26$ mag arcsec ${ }^{-2}$ and effective radius $>1.5 \mathrm{kpc}$ to the class of ultra-diffuse galaxies (UDGs). NGC 3669-DGSAT-3, the galaxy with the lowest surface brightness in our sample, may also be an UDG.
\end{abstract}

Key words. galaxies: spiral - galaxies: bulges - galaxies: dwarf - galaxies: statistics - galaxies: fundamental parameters - gravitation

\section{Introduction}

The framework of the standard model of cosmology (SMoC) explains the striking difference between baryonic and apparent dynamical mass of galaxies (e.g. Lelli et al. 2016) by introducing dark matter. According to this model, galaxies form in a hierarchical process with smaller dark matter halos merging to form more massive ones (e.g., Del Popolo 2014). While encounters between different halos may initially be hyperbolic, dynamical friction and dissipated energy leads to efficient coagulation. The massive dark halos of major host galaxies formed in this way do not only allow for high rotation velocities at large galactocentric radii but also contain a large number of subhalos, among them a notable fraction of dwarf galaxies. In view of the stochastic nature of galaxy encounters (but see Buck et al. 2015), individual infall histories, and the large number of objects involved, the spatial distribution of satellite galaxies should be spheroidal around the dominant host. And their number increases monotonically with circular velocity $V_{\text {rot }}$ (and thus mass) of the host dark matter halo (Moore et al. 1999; Kroupa et al. 2010; Klypin et al. 2011; Ishiyama et al. 2013, Kroupa 2015).
The alternative approach, also successfully modelling orbital motions in galaxies, does not modify the amount of matter by introducing a dominant dark component, but - based on baryonic matter alone - generalises instead gravity by introducing a scale invariant term when acceleration is below Milgrom's constant $\left(a_{0} \approx 3.8 \mathrm{pc} \mathrm{Myr}^{-2}\right.$; Milgrom 1983a,b, 2009; Famaey \& McGaugh 2012; see also Moffat 2006). A significant fraction of dwarf galaxies may then be of tidal origin, their formation being caused by hyperbolic encounters of larger galaxies (e.g. Yang et al. 2014). Such tidal dwarfs, if formed together in one encounter, should occupy a well defined, distinct region in sixdimensional phase space.

It is of utmost importance to differentiate between these two basic concepts, related to galaxy evolution and the nature of the gravitational potential. This can be done by observationally testing predictions related to small-scale structure, for example, by referring to the observable number of dwarf galaxies, their spatial distribution, and their distribution in phase space. For the standard model, the Local Group (LG) contains too few dwarf galaxies in comparison to the predicted number in the SMoC (e.g. Klypin et al. 1999; Simon \& Geha 2007) and dwarf galaxies are far from being distibuted isotropically around the 
Group's dominant members, the Andromeda galaxy (M 31) and the Milky Way. Instead, they show a highly flattened distribution around each of the galaxies, forming a vast polar structure (VPOS) around the Milky Way and an edge-on viewed plain around M 31. In addition, isolated dwarf galaxies of the LG may be confined to two additional narrow planes (e.g. Kroupa et al. 2005; Ibata et al. 2013; Pawlowski et al. 2013, 2015; Pawlowski 2016, and, for attempts of simulation, e.g., Ahmed et al. 2017).

While this clearly disagrees with predictions of the $\Lambda$ cold dark matter $(\Lambda \mathrm{CDM})$ standard model, these data only shed light onto a single group of galaxies. Whether our Local Group is an extreme outlier or whether the dwarf galaxy distribution of the Local Group is characterising the generic properties of the majority of groups in the local Universe is still an open question. Also, it is important to quantify the properties of satellite systems in the non-Newtonian framework. If these are tidal dwarfs, then they further constrain the statistics of galaxy encounters and thus cosmological models. Less sensitive and less complete studies of nearby galaxies outside the LG also suggest highly flattened distributions, such as those around M 81, NGC 3109, Cen A, and M 83 (Bellazzini et al. 2013; Chiboucas et al. 2013; Ibata et al. 2014; Müller et al. 2015, 2016, 2017b; Tully et al. 2015), but - given the deep implications for fundamental physics and recent less conclusive results (e.g. Blauensteiner et al. 2017; Müller et al. 2017a) - more observations are mandatory to obtain a clearer picture.

With this contribution we provide a catalogue of nearby disk galaxies, which are good candidates for probing the number and distribution of their satellites. Detailed aspects of the motivation are outlined in Sect. 2, source selection criteria are discussed in Sect. 3, and the sample is introduced in Sect. 4. We also present deep images from two of the sample galaxies in Sect. 5, followed by a short summary in Sect. 6 .

\section{Motivation}

A significant difference between the cosmological scenarios outlined above is related to the formation of bulges. If structure formation is governed by dark matter, a large number of galaxy mergers should possibly lead to the early formation of bulges in each major galaxy located at the centre of a massive dark matter halo, with bulge mass related to the mass of the dark matter halo (e.g. Weinzirl et al. 2009; Kormendy et al. 2010; but see also Avila-Reese et al. 2014). Galaxy encounters in generalised gravity models (e.g. Renaud et al. 2016) should be rarer and not all of these should lead to mergers, since an absence of dark matter leads to a lesser amount of dynamical friction, allowing for a larger number of hyperbolic flybys. As a consequence, there may be more major galaxies with masses similar to the Milky Way, which do not show a bulge at all, consistent with observations (Fisher \& Drory 2011; Kroupa 2015) and with the gradual decrease of the fraction of bulgeless sources in the full sample of disk galaxies during the last $\approx 7.5 \times 10^{9} \mathrm{yr}$ (Sachdeva 2013). Such bulgeless galaxies may never have undergone a significant interaction, leading to dwarf galaxy populations devoid of tidal ones or, referring to our Local Group where most dwarfs appear to be tidal (e.g. Pawlowski et al. 2015), to a few or even a total absence of dwarf galaxies. On the other hand, those galaxies having undergone significant encounters should show a (pseudo-)bulge, accompanied by at least one plane of tidal dwarfs, possibly rotating in the same sense.

As a consequence, with generalised gravity, dwarf galaxy populations of massive hosts with and without bulges should be very different. In the standard dark matter based model, even bulgeless massive disk galaxies with high rotation velocity $\left(V_{\text {rot }}\right)$ should have many satellites, while in generalised gravity models the number of dwarfs should be positively correlated with bulge mass.

Taking our Local Group as a template, where Andromeda (M 31), the Milky Way and the Triangulum galaxy (M 33) are the most prominent members, we find (Kroupa et al. 2010, their Fig. 3) a rising number of dwarf spheroidal satellite galaxies (dSph) with bulge mass. The correlation is consistent with a linear one, with M 33 (no bulge) hosting none and M 31 (most massive bulge) being surrounded by the largest number of dSphs. However, as already mentioned above with respect to the Local Group, these results are suggestive but can still be challenged on statistical grounds (see, e.g., Metz et al. 2009 and Libeskind et al. 2009, 2015). Furthermore, one may ask whether M 33 can be termed an independent galaxy or merely a large satellite of the Andromeda galaxy. More recently, López-Corredoira \& Kroupa (2016) found evidence for a positive correlation between the bulge/disk ratio of a major galaxy of given luminosity (from the Sloan Digital Sky Survey) and the number of associated tidal dwarf galaxies (from the catalogue of Kaviraj et al. 2012).

If the number of dwarf galaxies is found not to be related to bulge properties but instead to $V_{\text {rot }}$, this would weaken the argument that most dwarfs are of tidal origin. In any case, if we really want to provide basic constraints for cosmological models as well as the nature of gravity, the correlation between bulge mass and dwarf galaxy population has to be studied in much greater detail, requiring deep state of the art observations of a large number of galaxies, including a reliable determination of their distances.

\section{Selection criteria to find suitable targets}

To achieve this goal it is useful to compile a catalogue of suitable host galaxies. The most straightforward way is to focus on Milky Way-class edge-on $\mathrm{L}^{*}$ galaxies, where the presence or absence of a bulge is clearly seen and where a bulge mass can be derived. This is our first criterion. Such a sample with a single clear-cut viewing angle also allows for clear determinations of rotation curves. Furthermore, it will avoid pitfalls related to geometrical effects and will provide a detailed comparison not only between objects of different bulge mass but also between sources with similar bulge to disk masses or luminosities. This implies that also the scatter among objects with similar properties can be evaluated. Finally, the inclination of the potentially observed families of dwarf galaxies may reveal some scatter of angles with respect to the host's large scale plane, depending on the kind of interactions in the past.

Focusing on the environment of edge-on galaxies will greatly facilitate the determination of angles between their disks and potential planes of dwarf galaxies. Targets of choice should thus be those spiral galaxies, where an edge-on view is guaranteed. Then, for example, in case of a polar structure seen edge-on, the satellites would form a line similar to that shown by Kroupa et al. (2005; their Fig. 1) with significant shifts in radial velocity with respect to systemic at the outer edges $(V \sin \theta$, with $V$ denoting the rotation velocity and $\theta$ representing the angle of the line from the centre of the galaxy to the dwarf with respect to our line of sight). In case such a structure is seen face-on, satellites should show a rather isotropic distribution, but with no significant changes in radial velocity because of orbital motions being mostly confined within the plane of the sky. 
Because extremely deep exposures are required to detect as many dwarfs as possible (e.g. Merritt et al. 2014; Javanmardi et al. 2016), the field of view of optical telescopes is another important parameter. It should allow for direct imaging of the entire relevant volume surrounding a host galaxy. This is difficult to achieve. $\mathrm{L}^{*}$ disk galaxies are characterised by $M \approx 10^{12} M_{\odot}$ dark matter halos with virial radii $r_{\text {vir }} \approx$ $250 \mathrm{kpc}$ (e.g. Kravtsov 2013; Taylor et al. 2016), which must have grown from a sequence of mergers if the Standard Model of Cosmology is the valid theory. In the case of Andromeda, Perseus I, also called Andromeda XXXIII, is located at a distance of $375 \pm 15 \mathrm{kpc}$ from its host (Martin et al. 2013). Imaging projected radii out to $375 \mathrm{kpc}$, for a 0.5 field of view (FoV), distances $D \gtrsim 85 \mathrm{Mpc}$ and for a $3^{\circ} \mathrm{FoV}, D \gtrsim 15 \mathrm{Mpc}$ are required, corresponding to recession velocities of approximately $\gtrsim 12000$ and $\gtrsim 2000 \mathrm{~km} \mathrm{~s}^{-1}$, respectively. For a more modest projected diameter of $250 \mathrm{kpc}$, centred on a given host, 0.5 and $3^{\circ}$ FoVs require $D \gtrsim 30$ and $\gtrsim 4 \mathrm{Mpc}$, corresponding to recession velocities of $\gtrsim 2000$ and $\gtrsim 300 \mathrm{~km} \mathrm{~s}^{-1}$, respectively. Thus, field sizes well in excess of $1^{\circ}$ would be desirable.

Our second criterion, in clear opposition to the need for a coverage of large linear scales, is the requirement that the targets have to be spatially resolved. The detection of faint satellites is already difficult in the Local Group and much larger distances would lead to an overly high number of undetectable low-luminosity satellites. Resolving the galaxies is essential to prove that they are galaxies beyond doubt and to determine their surface brightness, effective radius $\left(r_{\mathrm{e}}\right)$, and magnitude. A characteristic $r_{\mathrm{e}}=500 \mathrm{pc}$ (e.g. Javanmardi et al. 2016) corresponds to $3^{\prime \prime}$ at a distance of $30 \mathrm{Mpc}\left(\approx 2000 \mathrm{~km} \mathrm{~s}^{-1}\right.$ recessional velocity; see also fig. 5 of Carrasco et al. 2006). This distance is close to the limit that could be reached by telescopes equipped with wide-field cameras and is, to provide a comparison, 2-5 times larger than the putative distances to the dwarf galaxy candidates identified by Merritt et al. (2014), Karachentsev et al. (2015), and Javanmardi et al. (2016).

The third criterion, again in opposition to the requirement of large linear scales to be covered simultaneously, is caused by field contamination due to unwanted foreground galaxies with their extended (on angular scales) foreground satellite systems. Thus again nearby galaxies are the targets of choice, while background sources covering substantially smaller solid angles may be recognised by their redshift (in case they are major galaxies) or by other properties (we refer to the end of this section and Sect. 5).

Finally, we have to avoid Galactic cirrus, which may inhibit a successful search for dwarfs often characterised by a low surface brightness (LSB). Therefore the galaxies should not be located close to the Galactic plane. This is the fourth criterion.

In the following section, we provide a list of suitable nearby edge-on targets, which are devoid of any nearby companions of similar angular size. As a caveat, we must note that although it is important to identify the dwarfs' association with their hosts beyond doubt, distances of such tiny galaxies would not be easy to obtain in a direct way (e.g. through brightest supergiant or tip of the red giant branch (TRGB) observations). Nevertheless, plots relating luminosity or surface brightness to effective radius or surface brightness to absolute magnitude (e.g. Javanmardi et al. 2016) can help to provide, beside a limited angular offset to the apparent host, significant first evidence for such an association (we refer to Sect. 5).

\section{The sample}

Among the sample of 1222 edge-on spiral galaxies in total from $\mathrm{NED}^{1}, 267$ are close enough to match our criterion of $V<2000 \mathrm{~km} \mathrm{~s}^{-1}$ (Sect. 3), which allows us to spatially resolve the objects but requires wide fields $(>0.5)$ of view. In addition, among the $55 \mathrm{~S} 0 / \mathrm{a}$ and $391 \mathrm{~S} 0$ galaxies, 12 and 89 are characterised by velocities $\leq 2000 \mathrm{~km} \mathrm{~s}^{-1}$, respectively. These 368 nearby sources were individually checked through images from NED and the SIMBAD Astronomical Database ${ }^{2}$. In some cases ratings of "very good" were assigned, while the remaining ones were discarded. Galaxies showing significant distortions were not included. Galaxies not exactly seen edge-on were downgraded. A frequently arising question was, whether an extended bulge was existing or the galaxy was instead not quite edge-on. These galaxies, mostly suffering from a limited image quality, were also excluded. In this way, 21 isolated edge-on targets could be identified.

A second sample has been drawn from the $\approx 5750$ sources presented by Bizyaev et al. (2014) in their catalogue of edge-on disk galaxies, based on the Sloan Digital Sky Survey (SDSS). Limiting the sample again to $V \leq 2000 \mathrm{~km} \mathrm{~s}^{-1}, 171$ sources were extracted and individually checked. Using the same procedure as for the NED objects, 22 were rated to be "very good". Not surpringly, there is some overlap with NED so that the number of additional sources is only 16 . Two of them, even though showing radial velocities close to $0 \mathrm{~km} \mathrm{~s}^{-1}$, are so faint that bulge properties could not be assigned. Excluding these two, which may show low intrinsic luminosity, that is PGC 1653789 and SDSS J081732.25+393629.0, then leads to $21+14=$ 35 galaxies.

Also applying the fourth criterion (Sect. 3) and requesting a sufficient angular distance from the Galactic plane, we find three sources with Galactic latitude $\left|b^{\mathrm{II}}\right|<15^{\circ}$. These were discarded, leaving a total of 32 targets. Among the latter, only NGC 891 is located at a $\left|b^{\mathrm{II}}\right|$ slightly below $20^{\circ}$. For all others, $\left|b^{\mathrm{II}}\right|>28^{\circ}$.

To summarise, the selected sub-sample is not free of observational bias, depending on the quality of the images and the brightness of the respective target. Thus, there may be suitable galaxies rejected by our harsh selection criteria. However, the goal of this article is not to present a complete sample but to guarantee that each selected galaxy is genuine. The best candidates are truly edge-on viewed bulgeless targets as well as galaxies with bulges, where either a dust lane partially obscuring the nuclear region clearly identifies the equatorial plane and/or where the outer disk is extremely thin, consistent with an edgeon view. Already a small number of such galaxies are sufficient to provide an answer to the questions raised in Sects. 1-3.

Table 1 presents the source list, consisting of 32 Milky Wayclass objects with $\log \left(L_{\mathrm{B}} / L_{\odot}\right)=8.6-11.1$. Infrared luminosities $L_{\mathrm{IR}}$ obtained by fitting IRAS data and extrapolating to the full range of 8-400 $\mu \mathrm{m}$ (for details, see Wouterloot \& Walmsley 1986) tend to be lower or close to the blue luminosities (exceptions: ESO 121-G006 and, see below, NGC 4634 with $L_{\mathrm{IR}} / L_{\mathrm{B}} \approx 3$ ). None of our objects is a vigorously active galaxy (with a recent starburst totally dominating $L_{\mathrm{B}}$ ), thus ensuring that our blue luminosities represent approximately the masses of the listed galaxies. Their distribution on the sky is shown in Fig. 1. Of our sources 11 exhibit at least some trace of an equatorial

1 The NASA/IPAC Extragalactic Database (NED) is operated by the Jet Propulsion Laboratory, California Institute of Technology, under contract with the National Aeronautics and Space Administration.

2 This research has made use of the SIMBAD database, operated at CDS, Strasbourg, France 
Table 1. The sample of nearby edge-on galaxies as targets of dedicated searches for associated dwarf galaxies.

\begin{tabular}{|c|c|c|c|c|c|c|c|c|}
\hline Name & $\alpha_{J 2000}$ & $\delta_{J 2000}$ & $l^{\mathrm{II}}$ & $b^{\mathrm{II}}$ & $\begin{array}{r}V \\
\left(\mathrm{~km} \mathrm{~s}^{-1}\right) \\
\end{array}$ & $\begin{array}{c}D \\
(\mathrm{Mpc})\end{array}$ & $\begin{array}{c}\log L_{\mathrm{B}} \\
\left(L_{\odot}\right)\end{array}$ & Bulge \\
\hline NGC 7814 & 000314.89 & +160843.5 & 106.4 & -45.2 & 1050 & $16 \pm 3$ & 10.2 & $\mathrm{p}+$ \\
\hline NGC 100 & 002402.98 & +162913.6 & 113.5 & -45.9 & 850 & $17 \pm 3$ & 9.1 & - \\
\hline UGC 1281 & 014931.61 & +323519.5 & 136.9 & -28.7 & 150 & $6 \pm 1$ & 8.6 & - \\
\hline ESO 477-G016 & 015615.99 & -225404.0 & 200.4 & -74.7 & 1825 & $36 \pm 5$ & 10.1 & $\mathrm{~m}$ \\
\hline NGC 891 & 022233.41 & +422056.9 & 140.4 & -17.4 & 525 & $10 \pm 1$ & 10.4 & $\mathrm{~m}+$ \\
\hline NGC 1886 & 052148.15 & -234836.5 & 226.3 & -29.6 & 1750 & $31 \pm 5$ & 9.8 & $m+$ \\
\hline ESO 121-G006 & 060729.86 & -614827.3 & 271.0 & -28.8 & 1200 & $20 \pm 3$ & 9.5 & $\mathrm{~s}$ \\
\hline NGC 2549 & 081858.35 & +574811.0 & 159.7 & +34.2 & 1075 & $17 \pm 7$ & 9.8 & $\mathrm{p}$ \\
\hline NGC 2654 & 084911.83 & +601316.2 & 156.1 & +37.8 & 1350 & $28 \pm 4$ & 10.0 & $\mathrm{~m}$ \\
\hline UGC 5245 & 094734.49 & -020157.1 & 238.8 & +37.0 & 1400 & $21 \pm 4$ & 9.0 & - \\
\hline NGC 3098 & 100216.69 & +244239.9 & 206.8 & +52.1 & 1400 & $20 \pm 6$ & 9.7 & $\mathrm{~m}$ \\
\hline NGC 3115 & 100513.98 & -074306.9 & 247.8 & +30.8 & 675 & $10 \pm 2$ & 10.3 & $\mathrm{p}$ \\
\hline UGC 5459 & 100810.07 & +530500.5 & 160.8 & +50.3 & 1100 & $22 \pm 4$ & 10.1 & -+ \\
\hline NGC 3669 & 112526.81 & +574316.2 & 143.3 & +55.9 & 1940 & $43 \pm 23$ & 10.2 & $\mathrm{~s}$ \\
\hline UGC 6792 & 114923.30 & +394617.4 & 164.5 & +72.0 & 850 & $20 \pm 3$ & 9.0 & - \\
\hline NGC 4179 & 121252.11 & +011758.9 & 281.6 & +62.6 & 1250 & $18 \pm 4$ & 9.9 & $\mathrm{~m}$ \\
\hline UGC 7321 & 121734.00 & +223224.5 & 241.9 & +81.1 & 400 & $18 \pm 6$ & 9.1 & - \\
\hline NGC 4244 & 121729.66 & +374825.6 & 154.6 & +77.2 & 250 & $04 \pm 2$ & 9.7 & $s+$ \\
\hline UGC 7394 & 122027.66 & +012811.1 & 285.5 & +63.3 & 1600 & $32 \pm 3$ & 9.2 & - \\
\hline NGC 4565 & 123620.78 & +255915.6 & 230.8 & +86.4 & 1225 & $12 \pm 4$ & 10.8 & $\mathrm{~m}+$ \\
\hline ESO 575-G061 & 130815.60 & -210008.0 & 308.2 & +41.7 & 1650 & $29 \pm 5$ & 9.4 & - \\
\hline NGC 5170 & 132948.79 & -175759.1 & 315.7 & +44.0 & 1500 & $27 \pm 7$ & 10.9 & $\mathrm{~s}$ \\
\hline UGC 8880 & 135711.48 & +502608.6 & 99.1 & +63.5 & 1850 & & & $\mathrm{~m}$ \\
\hline NGC 5470 & 140631.70 & +060141.0 & 346.5 & +62.4 & 1025 & $24 \pm 3$ & 9.2 & $\mathrm{~m}+$ \\
\hline UGC 9242 & 142521.02 & +3932 22.5 & 71.4 & +66.9 & 1475 & $20 \pm 6$ & 9.0 & - \\
\hline UGC 9249 & 142659.78 & +084101.0 & 358.2 & +60.9 & 1375 & $19 \pm 3$ & 8.9 & - \\
\hline NGC 5746 & 144455.92 & +015718.0 & 355.0 & +53.0 & 1725 & $30 \pm 3$ & 11.1 & $\mathrm{~m}+$ \\
\hline NGC 5866 & 150629.50 & +554547.6 & 92.0 & +52.5 & 750 & $12 \pm 4$ & 10.1 & $\mathrm{p}+$ \\
\hline NGC 5907 & 151553.77 & +561943.6 & 91.6 & +51.1 & 675 & $16 \pm 2$ & 10.8 & -+ \\
\hline UGC 9977 & 154159.54 & +004246.1 & 7.3 & +41.3 & 1925 & $29 \pm 3$ & 10.3 & -+ \\
\hline ESO 146-G014 & 221300.43 & -620403.4 & 328.6 & -46.4 & 1700 & $21 \pm 2$ & 9.4 & - \\
\hline IC 5176 & 221455.93 & -665057.9 & 323.0 & -43.7 & 1750 & $27 \pm 3$ & 10.3 & $\mathrm{~s}$ \\
\hline
\end{tabular}

Notes. Column (1): source name. Columns (2)-(5): equatorial and Galactic coordinates. Column (6): systemic velocity from NED, rounded to $25 \mathrm{~km} \mathrm{~s}^{-1}$. Column (7): here and elsewhere in the text, distances $D$ are average values from the literature given by NED. If there is no distance determination independent of radial velocity, no value is given. Column (8): the logarithm of the blue luminosity $\left(L_{\mathrm{B}}\right.$ in solar units) was taken from the "RC3" of de Vaucouleurs et al. (1991), correcting for inclination, internal extinction, and redshift. Only for NGC 1886, ESO 121-G006, and ESO 311-G012 were luminosities calculated from RC3 uncorrected blue magnitudes. Column (9): - indicates the apparent absence of a bulge; otherwise, with increasing dominance of a bulge, small $=\mathrm{s}$, moderate $=\mathrm{m}$, and prominent $=\mathrm{p}$ (see Sect. 4). A " + " indicates that at least some traces of an equatorial dust lane are present.

dust lane, providing a particularly reliable confirmation of an edge-on view. Of the 32 sources, 13 appear to be bulgeless. The remaining 19 targets have bulges of different prominence, ranging from small to prominent. The bulges are classified following their extent along the major axis of a given host relative to the total extent of the edge-on viewed disk and contain the following specifications:

Prominent: the bulge extends over $45 \%-85 \%$ of the length of the visible plane of the galaxy.

Moderate: the bulge covers $20 \%-<45 \%$ of the visible length of the host.

Small: an inconspicious bulge may be present.
Therefore, targets of all classes could be studied without contamination from other nearby sources. While the galaxies show no similarly sized nearby counterpart, the fields are nevertheless often not completely empty. Therefore, details of the environment of individual edge-on targets are discussed, source by source, in Appendix A.

While the presence of many Milky Way-sized galaxies in groups or clusters would lead to galaxy distributions which are too complex to analyse, the presence of two relatively nearby $\mathrm{L}^{*}$ objects may still not provide unsurmountable problems. Furthermore, the Local Group is based on a pair of two such galaxies, the Milky Way and M 31. It is by far the best studied galaxy group and naturally serves as THE template. Whether such pairs show a stochastic superposition of the dwarf galaxy populations 


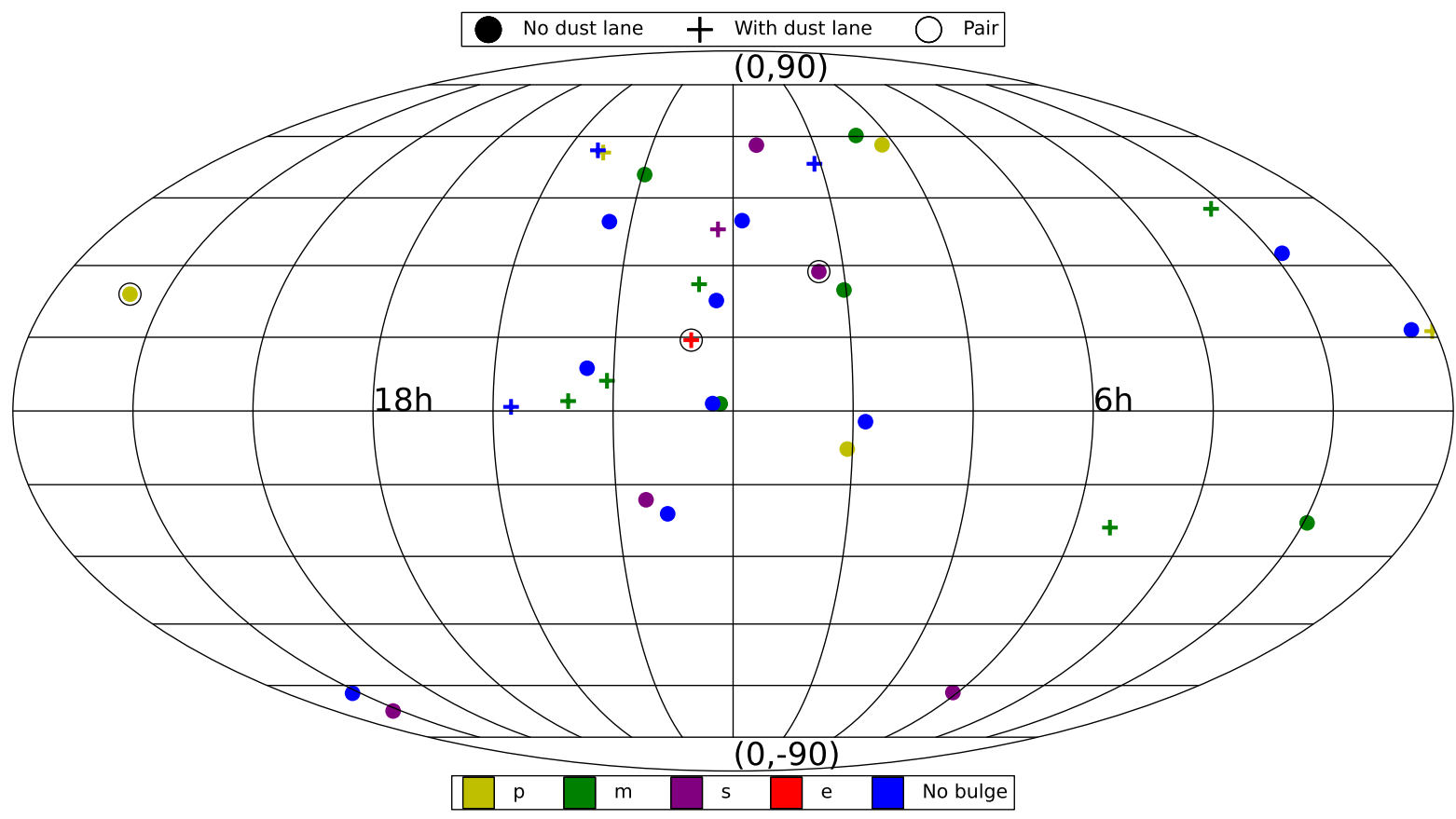

Fig. 1. Distribution of our sample of isolated galaxies (see Table 1) and galaxy pairs (Table 2) in J2000 equatorial coordinates on the sky. Pairs are indicate by an outer circle while the inner symbol refers to their edge-on member. Bulge sizes (see Sect. 4) and presence of equatorial dust lanes are also indicated.

Table 2. Nearby galaxy pairs.

\begin{tabular}{lccccccccc}
\hline \hline \multicolumn{2}{c}{ Names } & $\alpha_{J 2000}$ & $\delta_{J 2000}$ & $l^{\mathrm{II}}$ & $b^{\mathrm{II}}$ & $\begin{array}{c}V \\
\left(\mathrm{~km} \mathrm{~s}^{-1}\right)\end{array}$ & $\begin{array}{c}D \\
(\mathrm{Mpc})\end{array}$ & $\begin{array}{c}\log L_{\mathrm{B}} \\
\left(L_{\odot}\right)\end{array}$ & Bulge \\
\hline NGC 3245A & NGC 3245 & 102701.13 & +283821.6 & 201.6 & +58.2 & 1325 & $25 \pm 3$ & 9.8 & $\mathrm{~s}$ \\
NGC 4634 & NGC 4633 & 124240.96 & +141745.0 & 293.5 & +77.0 & 300 & $21 \pm 2$ & 9.4 & $\mathrm{e}+$ \\
NGC 7332 & NGC 7339 & 223724.54 & +234754.0 & 87.4 & -29.7 & 1200 & $15 \pm 6$ & 9.8 & $\mathrm{p}$ \\
& & & & & & & & &
\end{tabular}

Notes. Columns (1) and (2): names of the edge-on galaxy and its companion. All following parameters are those of the edge-on galaxy. NGC 3245 is an SA0 galaxy that is not seen edge-on, NGC 4633 is a spiral at intermediate and NGC 7339 a spiral at high inclination. Columns (3)-(6): equatorial and Galactic coordinates. Column (7): systemic velocity from NED. Column (8): distances $D$ are average values from the literature given by NED. For NGC 4634 the distance was obtained from its companion NGC 4633. We note, however, that this distance estimate is only based on two independent estimates. Column (9): the logarithm of the blue luminosity ( $L_{\mathrm{B}}$ in solar units) was taken from "RC3" (de Vaucouleurs et al. 1991), correcting for inclination, internal extinction, and redshift. Column (10): small bulge $=\mathrm{s}$, prominent bulge $=\mathrm{p}$ and boxy extended bulge $=$ e. + : at least some traces of an equatorial dust lane are present.

expected for individual parent galaxies or whether the distributions are correlated in some way as proposed by Libeskind et al. (2016) on the basis of SDSS data remains an open question. Potentially interesting in this context are the pairs NGC 3245 - NGC 3245A, NGC 4633 - NGC 4634, and NGC 7332 NGC 7339. Relevant data from the edge-on members of these pairs are presented in Table 2 and further information is given, as for the isolated Milky Way-sized targets, in Appendix A.

Finally, it is worth mentioning that the famous Sombrero Galaxy (M 104, distance $D=11 \pm 4 \mathrm{Mpc}$; Galactic latitude $b^{\mathrm{II}}$ $=+51 \% 1$ ) with its prominent bulge is not part of our sample because its inclination is not close enough to edge-on (the central core is not hidden by its dusty equatorial disk). Nevertheless, with an inclination of $i \approx 85^{\circ}$ (e.g. Bajaja et al. 1991), it is still close to edge-on and the galaxy is located in an "empty" field. For studies, which consider $i \approx 85^{\circ}$ as a sufficiently rigorous inclination limit, it is thus another host galaxy providing an interesting environment worthy of study (see Javanmardi et al. 2016).
Also interesting may be NGC 4631, the Whale Galaxy ( $D=$ $6 \pm 2 \mathrm{Mpc} ; b^{\mathrm{II}}=+84.2$ ), another edge-on galaxy with an equatorial dust lane but a smaller bulge (again, we refer to Javanmardi et al. 2016). It is not part of Table 2 because its overall shape appears to be asymmetric and distorted due to an interaction with NGC 4656, the Hockey Stick Galaxy. The latter is a nearby companion of only slightly lower luminosity with a morphology that is also affected by this interaction.

\section{New DGSAT data}

In the following we present deep images of two of the isolated galaxies in our catalogue (Table 1): NGC 3669 with a small bulge (Figs. 2 and 3) and NGC 7814 with a prominent bulge (Figs. 4 and 5), being part of the Dwarf Galaxy Survey with Amateur Telescopes (we refer to Javanmardi et al. 2016 for an introduction). Both of these images were obtained with the ROSA (POLLUX) 0.4-m Newton f/3.75 telescope containing a 


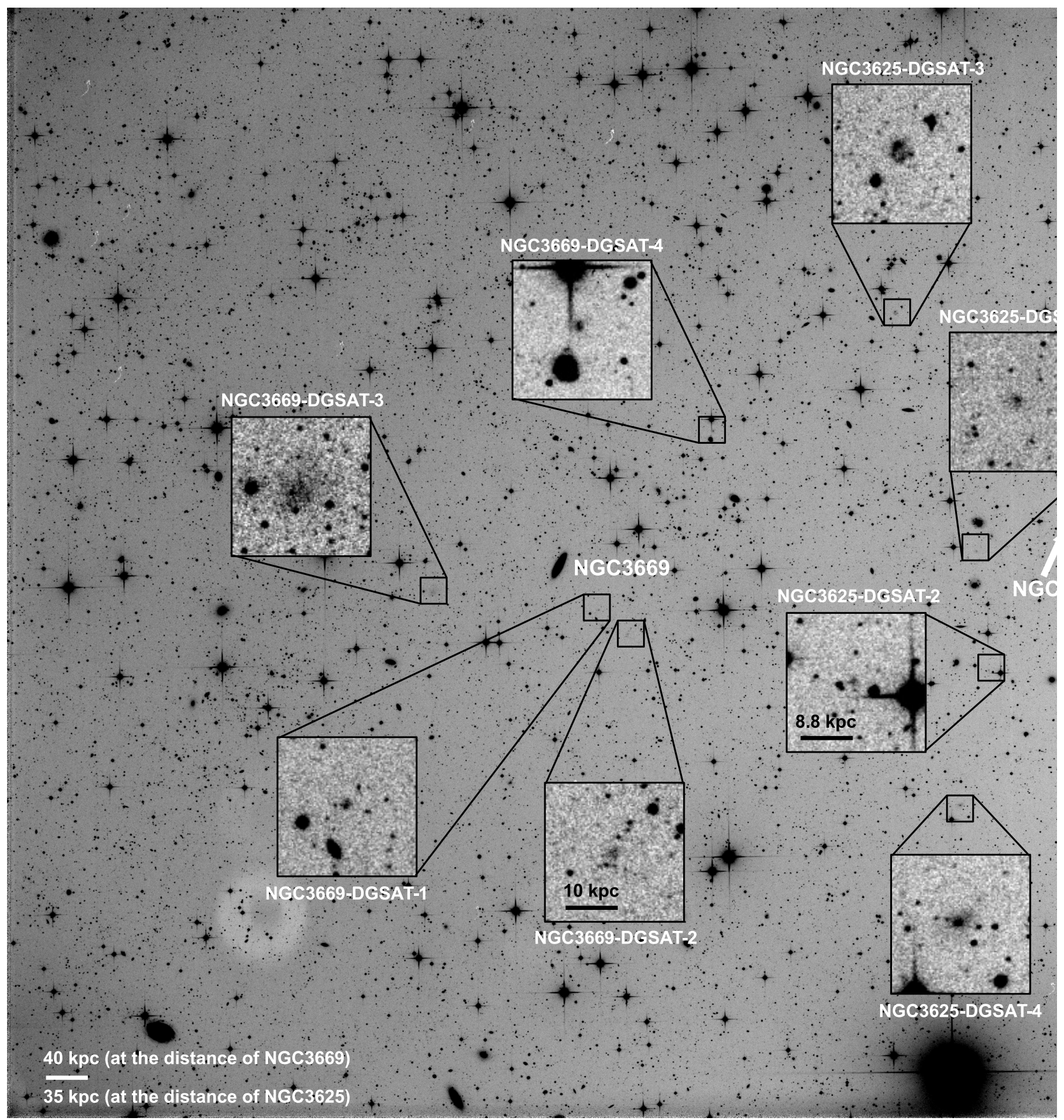

Fig. 2. A $1.4 \times 1.4$ image centred on NGC 3669 . North is up and east is to the left. The zoomed-in squares of size $101 \times 101$ pixels $\left(125^{\prime \prime} \times 125^{\prime \prime}\right)$ highlight the LSB galaxies in the image.

CCD camera covering a field of $\approx 1.4 \times 1.4$ with a pixel size of 1 '.24 and a full width at half maximum resolution of 2 '.78. Images were obtained with a wide band filter known as luminance (Astrodon Gen2 Tru-Balance E-series LRGB) that almost covers the entire SDSS $r$ and $g$ bands. NGC 3669 was observed in mid-December 2015 and mid-January 2016 with a total exposure time of $350 \mathrm{~min}$, while NGC 7814 was observed at the end of September 2016 for $400 \mathrm{~min}$.

The calibration of the images to SDSS $r$ band, the determination of the limiting surface brightness, the search for LSB galaxies and the extraction of their observed parameters was carried out in exactly the same way as in Javanmardi et al. (2016). Results referring to individual images are explained below.

\subsection{The NGC 3669 (small bulge) field}

The image of the field of NGC 3669 is shown in Fig. 2. The $5 \sigma$ limiting surface brightness is $27.7 \mathrm{mag} / \operatorname{arcsec}^{2}$ and the standard deviation of its calibration is $0.02 \mathrm{mag}$. We have detected eight LSB galaxies in the field of this image, which are also shown in Fig. 2. At the distance of NGC 3669, four of these LSB galaxies are within a projected distance of approximately $200 \mathrm{kpc}$ to NGC 3669, while the other four are off by $\approx 400 \mathrm{kpc}$ or more. The latter four are (in projection) closer to NGC 3625 (which is located near the western edge of our image), and, therefore, are named after this galaxy. Seven of these eight LSB galaxies have already been detected by the SDSS and are catalogued as 

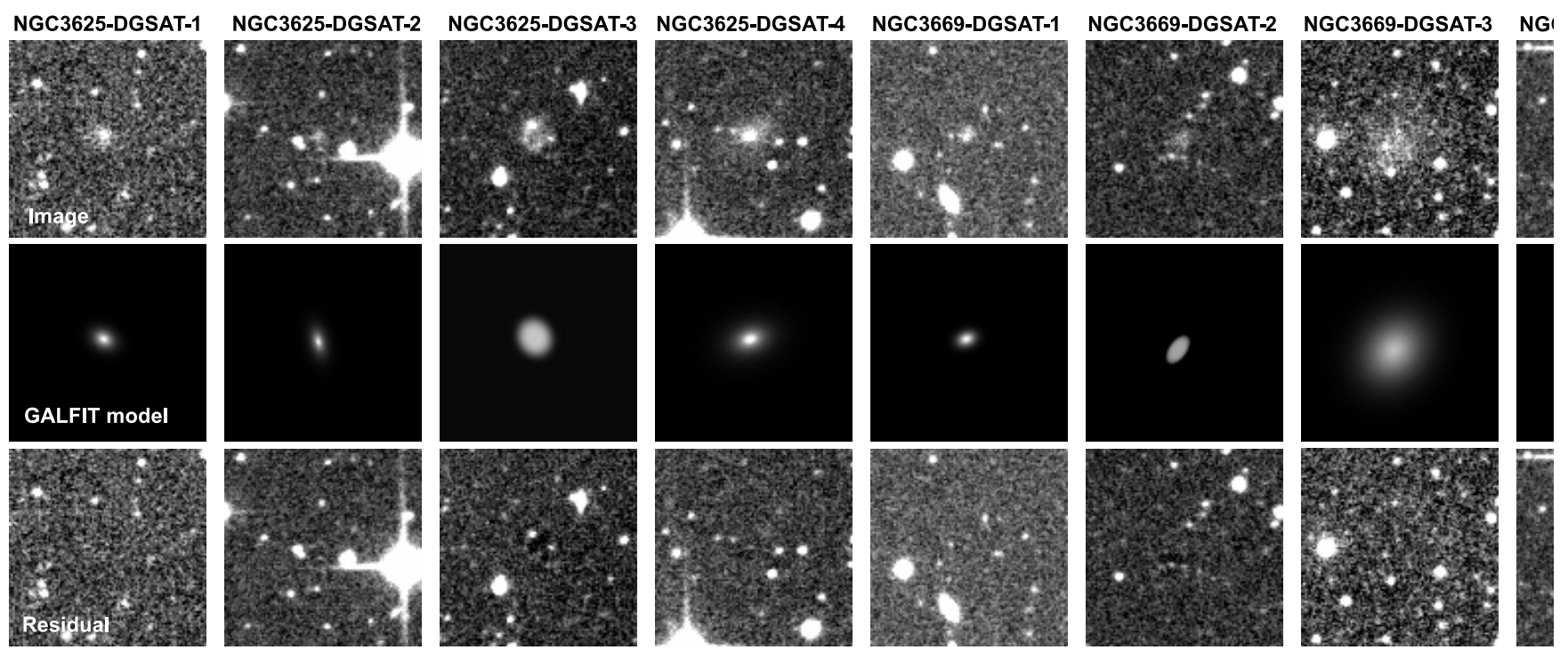

Fig. 3. Top row: dwarf galaxy candidates detected in our image; middle row: our GALFIT models (see Table 3 for the results of the modelling), and bottom row: residual images obtained by subtracting the model image (middle) from the main image (top). As the inserts in Fig. 2, the images have a size of $101 \times 101$ pixels, corresponding to $125^{\prime \prime} \times 125^{\prime \prime}$. North is up and east is to the left.

Table 3. Observed parameters of identified LSB galaxies.

\begin{tabular}{cccccccc}
\hline \hline ID & $\alpha_{J 2000}$ & $\delta_{J 2000}$ & $r_{\text {cal }}(\mathrm{mag})$ & $\mu_{\mathrm{e}}\left(\mathrm{mag} / \mathrm{arcsec}^{2}\right)$ & $n$ & $R_{\mathrm{e}}$ (arcsec) & $b / a$ \\
\hline NGC 3625-DGSAT-1 & 112128.45 & +574355.2 & $19.9 \pm 0.1$ & $26.2 \pm 0.1$ & $0.83 \pm 0.08$ & $6.3 \pm 0.5$ & $0.78 \pm 0.04$ \\
NGC 3625-DGSAT-2 & 112122.91 & +573450.1 & $20.1 \pm 0.3$ & $26.7 \pm 0.3$ & $1.04 \pm 0.23$ & $8.5 \pm 1.9$ & $0.53 \pm 0.06$ \\
NGC 3625-DGSAT-3 & 112212.03 & +580211.9 & $18.8 \pm 0.1$ & $25.3 \pm 0.1$ & $0.26 \pm 0.02$ & $8.2 \pm 0.2$ & $0.87 \pm 0.02$ \\
NGC 3625-DGSAT-4 & 112140.79 & +572437.0 & $18.3 \pm 0.1$ & $26.1 \pm 0.1$ & $1.24 \pm 0.07$ & $12.0 \pm 0.8$ & $0.70 \pm 0.02$ \\
\hline NGC 3669-DGSAT-1 & 112504.39 & +573953.6 & $20.2 \pm 0.1$ & $25.8 \pm 0.1$ & $0.66 \pm 0.07$ & $5.1 \pm 0.4$ & $0.73 \pm 0.04$ \\
NGC 3669-DGSAT-2 & 112448.32 & +573758.0 & $19.8 \pm 0.1$ & $26.0 \pm 0.1$ & $0.19 \pm 0.03$ & $8.9 \pm 0.3$ & $0.56 \pm 0.02$ \\
NGC 3669-DGSAT-3 & 112638.78 & +574119.1 & $18.1 \pm 0.1$ & $26.7 \pm 0.1$ & $0.65 \pm 0.04$ & $18.6 \pm 0.9$ & $0.83 \pm 0.03$ \\
NGC 3669-DGSAT-4 & 112357.86 & +575325.6 & $19.3 \pm 0.1$ & $25.7 \pm 0.1$ & $0.83 \pm 0.05$ & $6.3 \pm 0.3$ & $0.82 \pm 0.03$ \\
\hline NGC 7814-DGSAT-1 & 00324.07 & +161111.1 & $17.7 \pm 0.1$ & $26.0 \pm 0.1$ & $0.59 \pm 0.03$ & $15.8 \pm 0.7$ & $0.86 \pm 0.03$ \\
NGC 7814-DGSAT-2 & 00306.93 & +161830.8 & $17.7 \pm 0.1$ & $26.7 \pm 0.1$ & $1.04 \pm 0.05$ & $29.4 \pm 1.5$ & $0.35 \pm 0.01$ \\
NGC 7814-DGSAT-3 & 00411.75 & +163201.2 & $18.1 \pm 0.1$ & $25.5 \pm 0.1$ & $0.78 \pm 0.03$ & $11.6 \pm 0.3$ & $0.62 \pm 0.01$ \\
NGC 7814-DGSAT-4 & 00248.89 & +163550.9 & $18.7 \pm 0.1$ & $25.8 \pm 0.1$ & $0.78 \pm 0.06$ & $8.5 \pm 0.5$ & $0.92 \pm 0.04$ \\
NGC 7814-DGSAT-5 & 00235.21 & +164253.4 & $20.6 \pm 0.1$ & $25.7 \pm 0.1$ & $0.39 \pm 0.07$ & $4.7 \pm 0.3$ & $0.62 \pm 0.04$ \\
NGC 7814-DGSAT-6 & 00027.94 & +161100.6 & $18.8 \pm 0.1$ & $24.9 \pm 0.1$ & $0.80 \pm 0.04$ & $5.9 \pm 0.2$ & $0.72 \pm 0.02$ \\
NGC 7814-DGSAT-7 & 00043.95 & +152714.3 & $18.5 \pm 0.1$ & $26.8 \pm 0.1$ & $1.14 \pm 0.11$ & $19.1 \pm 2.1$ & $0.49 \pm 0.02$ \\
\hline
\end{tabular}

Notes. Column (1): LSB galaxy. Columns (2) and (3): equatorial J2000 coordinates. Columns (4)-(8): structural parameters evaluated by GALFIT modelling (see Peng et al. 2002 and Javanmardi et al. 2016). Column (4): calibrated integrated magnitude, $r_{\text {cal }}$; Column (5): surface brightness at effective radius, $\mu_{\mathrm{e}}$; Column (6): sersic index, $n$; Column (7): effective radius, $R_{\mathrm{e}}$; Column (8): axis ratio, $b / a$.

galaxies, but all of them with low quality measurement flags. The remaining one, NGC 3669-DGSAT-3, is a particularly low surface brightness galaxy which appears to be very extended. We obtain the observed structural parameters of all eight galaxies by modelling them with a Sersic profile using the GALFIT software. The results are shown in Table 3 and Fig. 3.

\subsection{The NGC 7814 (prominent bulge) field}

Our image of the field of NGC 7814 is shown in Fig. 4. The $5 \sigma$ limiting surface brightness is $27.8 \mathrm{mag} / \operatorname{arcsec}^{2}$ and the standard deviation of its calibration is $0.01 \mathrm{mag}$. Seven LSB galaxies were detected in this image which are also shown in Fig. 4. The discovery of two of them, namely NGC 7814-DGSAT-1 and 2, has already been reported in the first DGSAT paper (Javanmardi et al. 2016). Out of the remaining five, four are catalogued by SDSS with low quality measurement flags. Our newly identified galaxy, NGC 7814-DGSAT-7, has the lowest surface brightness among our detections. Modelling results of all seven galaxies are shown in Table 3 and Fig. 5.

\subsection{Implications}

Our detected objects are characterised by a LSB, $\approx 25-$ $27 \mathrm{mag} / \mathrm{arcsec}^{2}$, cover a range of integrated magnitudes from $r_{\text {cal }}=17.7$ to $20.6 \mathrm{mag}$, and exhibit effective radii of order $10^{\prime \prime}$. Sersic indices are around unity or smaller, consistent with what is expected from small galaxies (e.g. Merritt et al. 2014). Subtracting the modelled galaxies from the original images (we refer to the lowest rows of images in Figs. 3 and 5), there are no residuals above the noise level. Thus no major irregularities, hinting at galaxy interactions, are found. Table 4 provides projected distances of our dwarfs to their putative hosts. Only three galaxies show values $<60 \mathrm{kpc}$, with NGC $7814-D G S A T-1$ at 


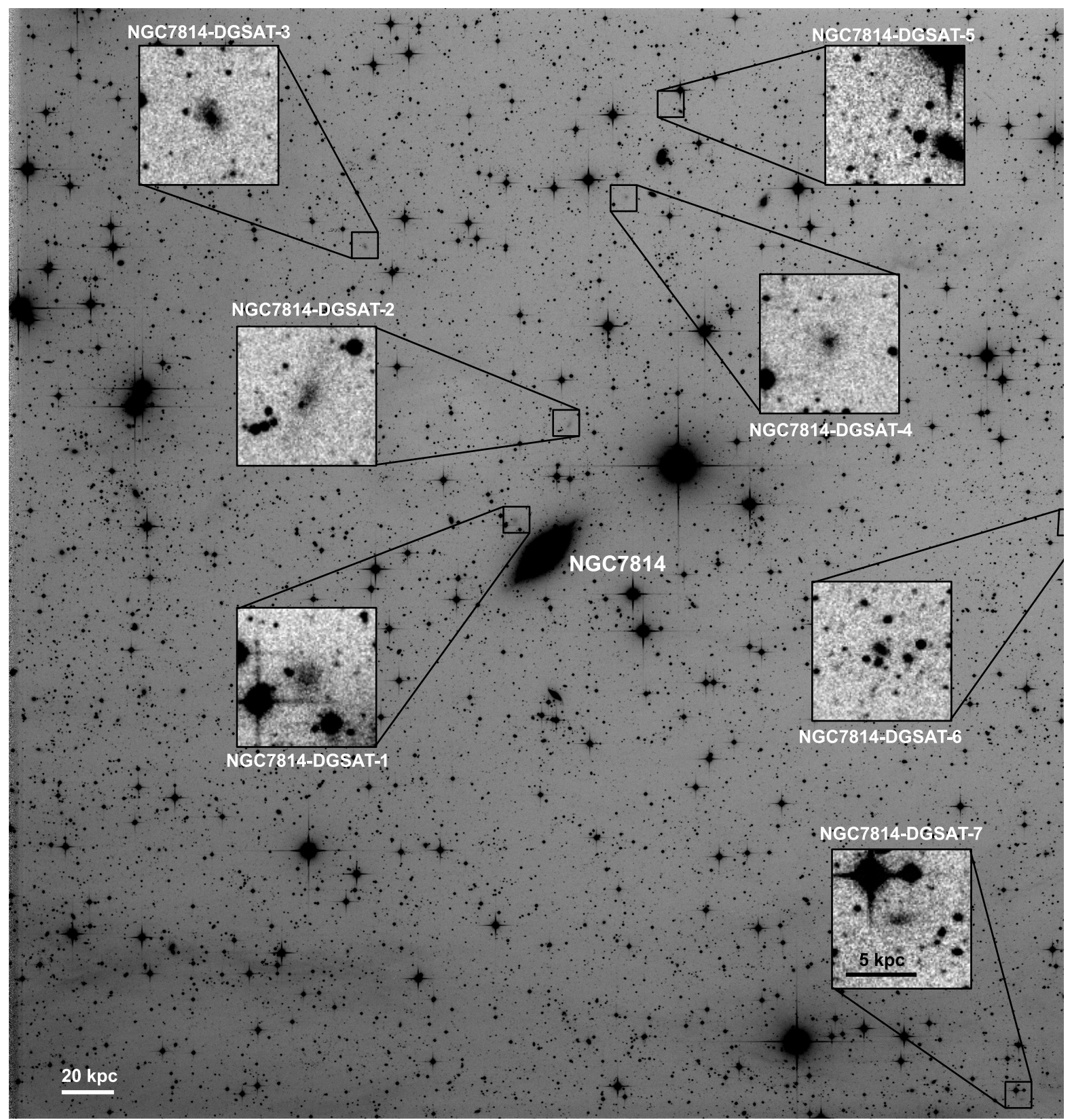

Fig. 4. The $1.4 \times 1.4$ field of NGC 7814. North is up and east is to the left. The zoomed-in squares highlight the LSB galaxies in the image. The angular size of the inserts is the same as in Fig. 2.

$\approx 16 \mathrm{kpc}$ representing the most critical case. That we see even here no significant distortion indicates, that its real distance must be larger. Only one of the targets, previously (Javanmardi et al. 2016) detected, NGC 7814-DGSAT-2, shows a minor-to-major axis ratio clearly below 0.5 .

Low surface brightness galaxies are difficult targets for follow-up measurements, trying to relate them beyond doubt with their major host. To provide support for such an association, in addition to their close line-of-sight positions, we therefore use the relations already mentioned at the end of Sect. 3. Data are displayed in Fig. 6, together with the known dwarf galaxies of the Local Group (McConnachie 2012).

Obviously, the galaxies characterised in this article (as those in Javanmardi et al. 2016) are following the correlations established for the Local Group galaxies with respect to the connections between luminosity and effective radius, effective radius and surface brightness, and absolute magnitude versus surface brightness. Our galaxies are located at the luminous and extended end of these correlations. This is an expected effect since analogues of less luminous LG dwarfs are not detectable at the distances to NGC 3669 (43 $\pm 23 \mathrm{Mpc})$, NGC 3625 $(37 \pm 4 \mathrm{Mpc})$ and NGC $7814(16 \pm 3) \mathrm{Mpc}$. Therefore associating our dwarfs with their putative massive hosts, we find absolute magnitudes of $M_{r}=-10.4$ to -14.8 (Table 4 ). In this context we should also note that NGC 3625 and NGC 3669 are located at larger distances than the galaxies studied by Karachentsev et al. (2014), Merritt et al. (2014), Javanmardi et al. (2016) or Müller et al. (2017a,b). Adopting the distances to the nearby major galaxies, NGC 3625-DGSAT-4, with a suface brightness much fainter than 24 mag $\operatorname{arcsec}^{-2}$ and a size $R_{\mathrm{e}}$ $>1.5 \mathrm{kpc}$, can be classified as an ultra-diffuse galaxy (see Merritt et al. 2016). NGC 3669-DGSAT-3, our galaxy with the lowest surface brightness, likely also belongs to this class, in spite of its highly uncertain effective radius. 

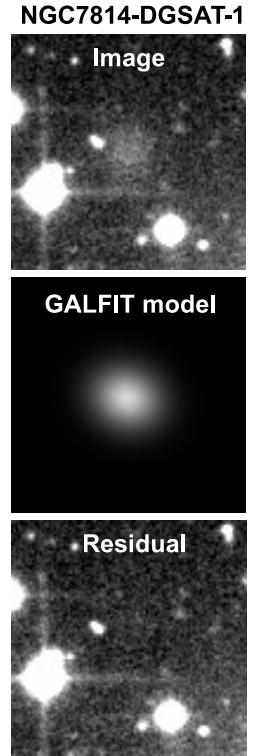
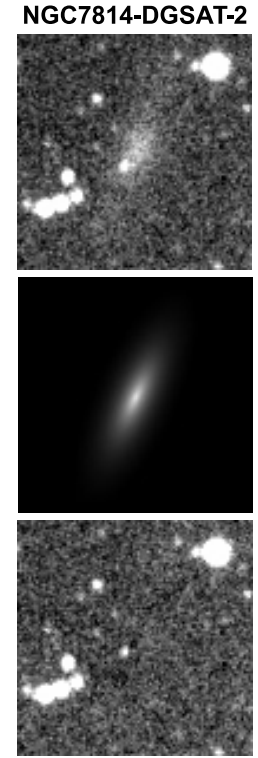
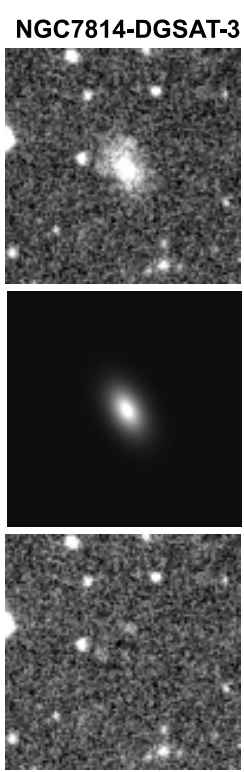
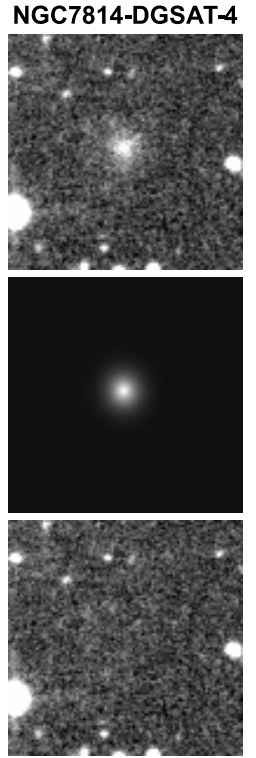
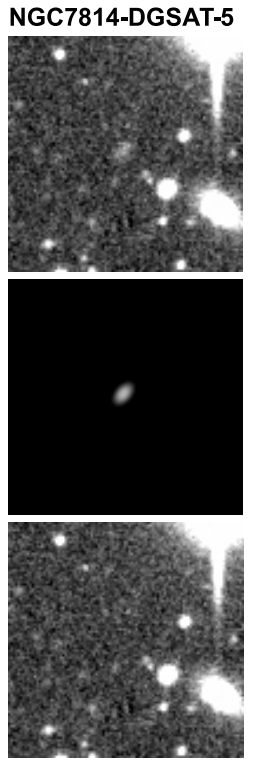

NGC7814-DGSAT-6 NGC7814-DGSAT-7
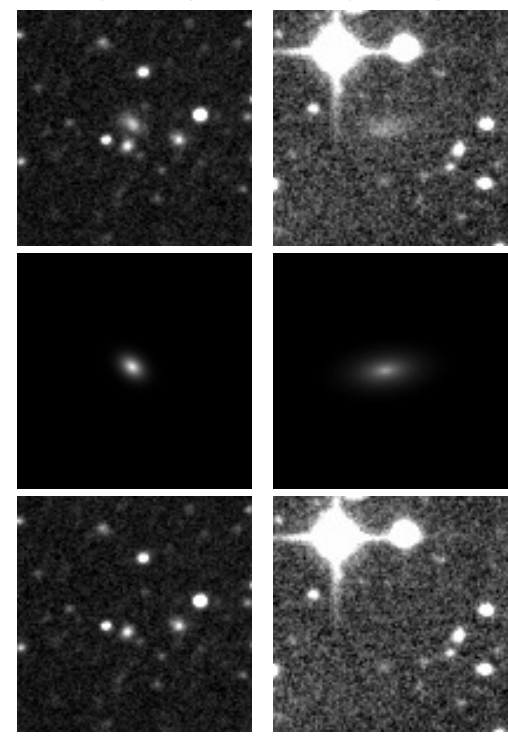

Fig. 5. Top row: dwarf galaxy candidates detected in our image; middle row: our GALFIT models (see Table 3 for the results of the modelling), and bottom row: residual images obtained by subtracting the model image (middle) from the main image (top). Orientation and angular size of the images are the same as in Fig. 3.

Table 4. Physical properties of the LSB galaxies assuming that they are satellites of the nearby (in projection) massive galaxies.

\begin{tabular}{lllll}
\hline \hline ID & $R_{\mathrm{e}}(\mathrm{pc})$ & $d_{\mathrm{P}(\mathrm{kpc})}$ & $M_{r}(\mathrm{mag})$ & $\log \left(\frac{L}{L_{\odot}}\right)_{r}$ \\
\hline NGC 3625-DGSAT-1 & $1145 \pm 156$ & $89.6 \pm 9.8$ & $-13.0 \pm 0.2$ & $7.1 \pm 0.1$ \\
NGC 3625-DGSAT-2 & $1536 \pm 380$ & $151.2 \pm 16.6$ & $-12.8 \pm 0.4$ & $7.0 \pm 0.2$ \\
NGC 3625-DGSAT-3 & $1480 \pm 166$ & $220.6 \pm 24.2$ & $-14.1 \pm 0.2$ & $7.5 \pm 0.1$ \\
NGC 3625-DGSAT-4 & $2175 \pm 275$ & $262.7 \pm 28.9$ & $-14.5 \pm 0.2$ & $7.7 \pm 0.1$ \\
\hline NGC 3669-DGSAT-1 & $1064 \pm 572$ & $57.4 \pm 30.6$ & $-12.7 \pm 1.2$ & $7.0 \pm 0.5$ \\
NGC 3669-DGSAT-2 & $1864 \pm 996$ & $93.0 \pm 49.5$ & $-13.1 \pm 1.2$ & $7.1 \pm 0.5$ \\
NGC 3669-DGSAT-3 & $3912 \pm 2094$ & $123.1 \pm 65.6$ & $-14.8 \pm 1.2$ & $7.8 \pm 0.5$ \\
NGC 3669-DGSAT-4 & $1325 \pm 709$ & $196.6 \pm 104.8$ & $-13.6 \pm 1.2$ & $7.3 \pm 0.5$ \\
\hline NGC 7814-DGSAT-1 & $1231 \pm 269$ & $15.6 \pm 3.4$ & $-13.2 \pm 0.5$ & $7.2 \pm 0.2$ \\
NGC 7814-DGSAT-2 & $2285 \pm 504$ & $46.7 \pm 10.0$ & $-13.3 \pm 0.5$ & $7.2 \pm 0.2$ \\
NGC 7814-DGSAT-3 & $901 \pm 195$ & $126.4 \pm 27.1$ & $-12.9 \pm 0.5$ & $7.0 \pm 0.2$ \\
NGC 7814-DGSAT-4 & $662 \pm 147$ & $129.8 \pm 27.9$ & $-12.3 \pm 0.5$ & $6.8 \pm 0.2$ \\
NGC 7814-DGSAT-5 & $366 \pm 82$ & $165.5 \pm 35.5$ & $-10.4 \pm 0.5$ & $6.0 \pm 0.2$ \\
NGC 7814-DGSAT-6 & $461 \pm 100$ & $187.6 \pm 40.3$ & $-12.1 \pm 0.5$ & $6.7 \pm 0.2$ \\
NGC 7814-DGSAT-7 & $1490 \pm 360$ & $257.9 \pm 55.4$ & $-12.5 \pm 0.5$ & $6.9 \pm 0.2$ \\
\hline
\end{tabular}

Notes. $R_{\mathrm{e}}$ and $d_{\mathrm{P}}$ are effective radius and projected distance of the dwarf candidate to the centre of the host galaxy, respectively. $M_{r}$ denotes the absolute magnitude in $r$-band and the last column provides the logarithm of the $r$-band luminosity in units of solar luminosities, $L_{\odot}$.

A final comment refers to the projected distribution of our LSB galaxies around their putative major hosts. In Sects. 1-3, we outlined potential distributions. For our galaxies, no obvious trend is yet apparent. Likely, the number of identified dwarfs is still too small (we refer to, for a related case, Müller et al. 2015, 2016, and 2017a and their analyses of the Centaurus group), emphasising the urgent need for even more sensitive follow-up studies.

\section{Conclusions}

As shown, there exists a number of promising major edge-on galaxy hosts with well determined bulge sizes, where dwarf galaxy distributions can be studied under geometrically advantageous conditions. The galaxies in so-called "empty" fields provided by our catalogue (Tables 1 and 2) will allow for efficient dwarf galaxy surveys of regions with radius $\gtrsim 250 \mathrm{kpc}$ (1) to test whether or not such dwarfs exist at all, (2) to search for the existence of a number of satellites - bulge mass/luminosity relation and (3) to find evidence for anisotropic satellite populations. In addition to isolated hosts in such fields, our sample also contains members of galaxy pairs, which have the potential to contribute significantly to our understanding of more complex galaxy configurations as they are, for example, encountered in our Local Group.

There are six arguments in support of a close connection of our low surface brightness (LSB) galaxies with the Milky Waysized galaxies in their projected neighbourhood:

- Our targets are, projected onto the plane of the sky, close to their putative host galaxies.

- Our targets are characterized by very low surface brightness.

- Sersic indices are around unity or smaller. 

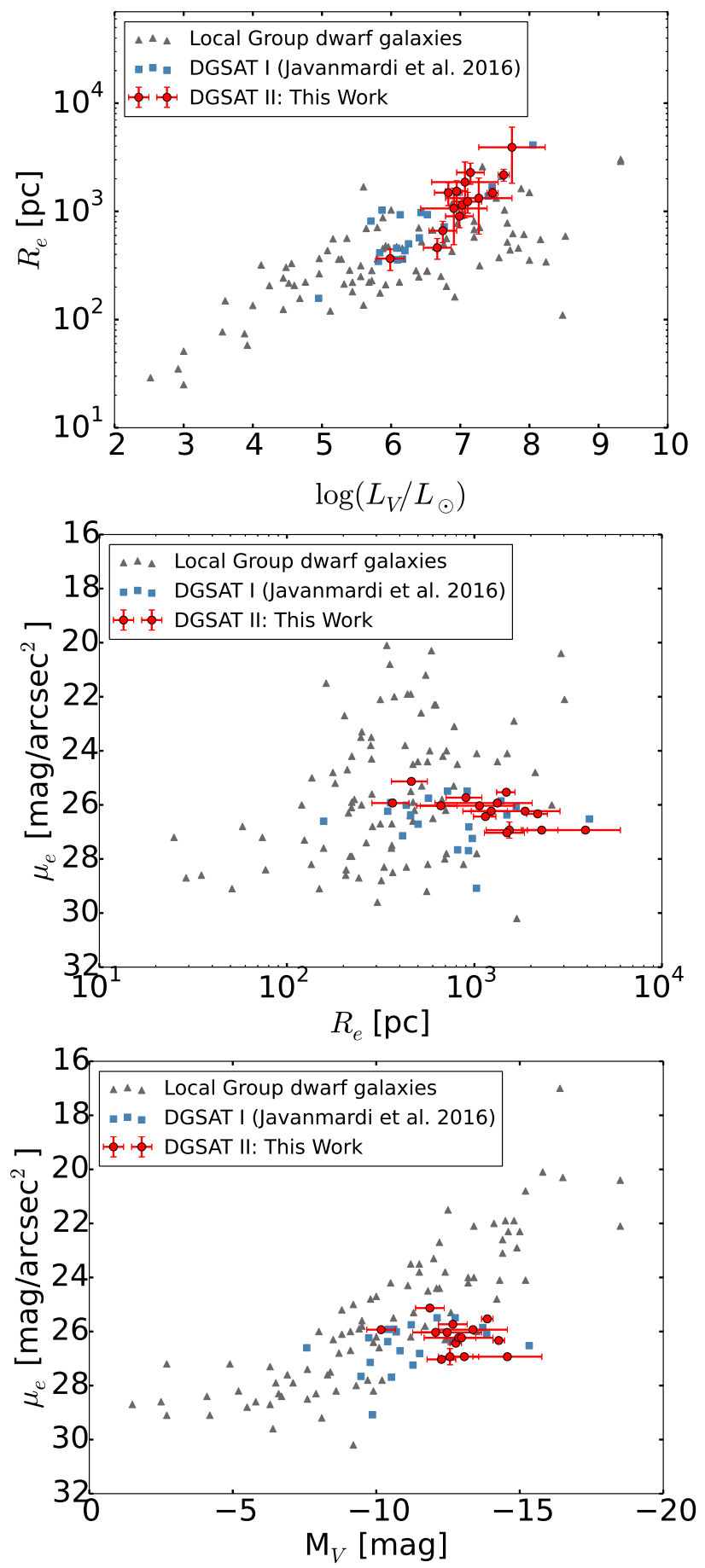

Fig. 6. A comparison between the properties of the dwarf galaxy candidates studied in this work, the first DGSAT results (Javanmardi et al. 2016), and the known dwarf galaxies of the Local Group (McConnachie 2012). Top: effective radius in pc versus logarithm of $V$ band luminosity in $L_{\odot}$. Middle: $V$ band surface brightness in mag/arcsec ${ }^{2}$ versus effective radius in pc. Bottom: $V$ band surface brightness in mag/ $\operatorname{arcsec}^{2}$ versus absolute $V$ band magnitude. The $V$ band quantities were obtained by transforming $r$ band magnitudes to $V$ magnitudes using Fukugita et al. (1996).

- Assuming that our LSB galaxies are at the distance of their supposed main group members, their correlation between effective radius and luminosity is consistent with that of Local Group dwarfs, being located at their luminous, extended end.
- This also holds for the correlation between effective radius and surface brightness.

- Finally, absolute magnitudes and surface brightnesses are also consistent with the assumption that our galaxies are located in the vicinity of their major galaxy hosts.

Concerning the bulge mass versus number of satellites correlation (see Kroupa et al. 2010; López-Corredoira \& Kroupa 2016), the results obtained here are four satellite candidates for NGC 3669 (small bulge) and seven candidates for NGC 7814 (prominent bulge). Right now, the existence of the relation can neither be excluded nor verified with high confidence and more observations are mandatory.

Acknowledgements. We are grateful for very good and useful referee comments. B.J. would also like to thank the Bonn-Cologne Graduate School (BCGS) for Physics and Astronomy for their financial support, and also R. GonzalezLopezlira, Z. Shafiee, and Z. Sheikhbahaee for useful discussions on image analysis. D.M-D. acknowledges support by the Sonderforschungsbereich (SFB) 881 "The Milky Way System" of the Deutsche Forschungsgemeinschaft (DFG), in particular through subproject A2. The SAO/NASA Astrophysical Data System (ADS) was very helpful when searching for relevant articles.

\section{References}

Ahmed, S. H., Brooks, A. M., \& Christensen, C. R. 2017, MNRAS, 466, 3119 Avila-Reese, V., Zavala, J., \& Lacerna, I. 2014, MNRAS, 441, 417

Bajaja, E., Krause, M., Wielebisnki, R., \& Dettmar, R.-J. 1991, A\&A, 241, 411 Bellazzini, M., Oosterloo, T., Fraternali, F., \& Beccari, G. 2013, A\&A, 559, L11

Bizyaev, D. V., Kautsch, S. J., Mosenkov, A. V., et al. 2014, ApJ, 787, 24 Blauensteiner, M., Karachentsev, I. D., Remmel, P., et al. 2017, arXiv e-print [arXiv: 1701.00615]

Buck, T., Macciò, A. V., \& Dutton, A. A. 2015, ApJ, 809, 49

Carrasco, E. R., Mendes de Oliveira, C., \& Infante, L. 2006, AJ, 132, 1796

Chiboucas, K., Jacobs, B. A., Tully, R. B., \& Karachentsev, I. D. 2013, AJ, 146, 126

Del Popolo, A. 2014, Int. J. Mod. Phys. D, 23, 1430005

de Vaucouleurs, G., de Vaucouleurs, A., Corwin, H. G., et al. 1991, 3rd Reference Catalogue of Bright Galaxies (RC3) (New York: Springer Verlag) Famaey, B., \& McGaugh, S. S. 2012, Living Rev. Relativity, 15, 10 Fisher, D.B., \& Drory, N. 2011, ApJ, 733, L47

Fukugita, M., Ichikawa, T., Gunn, J. E., et al. 1996, AJ, 111, 1748 Ibata, R. A., Lewis, G. F., Conn, A. R., et al. 2013, Nature, 493, 62 Ibata, N. G., Ibata, R. A., Famaey, B., \& Lewis, G. F. 2014, Nature, 511, 563 Ishiyama, T., Rieder, S., Makino, J., et al. 2013, ApJ, 767, 146 Javanmardi, B., Martínez-Delgado, D., Kroupa. P., et al. 2016, A\&A, 588, A89 Karachentsev, I. D., Riepe, P., Zilch, T. et al. 2015, Astrophys. Bull., 70, 379 Kaviraj, S., Darg, D., Lintott, C., Schawinski, K., \& Silk, J. 2012, MNRAS, 419. 70

Klypin, A., Kravtsov, A. V., Valenzuela, O., \& Prada, F. 1999, ApJ, 522, 82

Klypin, A. A., Trujillo-Gomez, S., \& Primack, J. 2011, ApJ, 740, 102 Kormendy, J., Drory, N., Bender, R., \& Cornell, M. E. 2010, ApJ, 723, 54 Kravtsov, A. V. 2013, ApJ, 764, L31

Kroupa, P. 2015, Can. J. Phys., 93, 169

Kroupa, P., Theis, C., \& Boily, C. M. 2005, A\&A. 431, 517

Kroupa, P., Famaey, B., de Boer, K. S., et al. 2010, A\&A 523, A32

Lelli, F., McGaugh, S. S., Schombert, J. M., \& Pawlowski, M. S. 2016, ApJ, 827, L19

Libeskind, N. I., Frenk, C. S., Cole, S., Jenkins, A., \& Helly, J. C. 2009, MNRAS, 399,550

Libeskind, N. I., Hoffmann, Y., Tully, R. B., et al. 2015, MNRAS, 452, 1052

Libeskind, N. I., Guo, Q., Tempel, E., \& Ibata, R. 2016, ApJ, 830, 121

López-Corredoira, M., \& Kroupa, P. 2016, ApJ, 817, 75

Martin, N. F., Schlafly, E. F., Slater, C. T., et al. 2013, ApJ, 779, L10

McConnachie, A. W. 2012, AJ, 144, 4

Merritt, A., van Dokkum, P., \& Abraham, R. 2014, ApJ, 787, L37

Merritt, A., van Dokkum, P., \& Danieli, S., et al. 2016, ApJ, 833, 168

Metz, M., Kroupa, P., Theis, C., Hensler, G., \& Jerjen, H. 2009, ApJ, 697, 269

Milgrom, M. 1983a, ApJ, 270, 365

Milgrom, M. 1983b, ApJ, 270, 371

Milgrom, M. 2009, ApJ, 698, 1630

Moffat, J. W. 2006, JCAP, 3, 4 
C. Henkel et al.: An edge-on sample of nearby galaxies

Moore, B., Ghigna, S., Governato, F., et al. 1999, ApJ, 524, L19

Müller, O., Jerjen, H., \& Binggeli, B. 2015, A\&A, 583, A79

Müller, O., Jerjen, H., Pawlowski, M.S., \& Binggeli, B. 2016, A\&A, 595, A119

Müller, O., Jerjen, H., \& Binggeli, B. 2017a, A\&A 597, A7

Müller, O., Scalera, R., Binggeli, B., \& Jerjen, H. 2017b, A\&A, in press, DOI: 10.1051/0004-6361/201730434

Pawlowski, M. S. 2016, MNRAS, 456, 448

Pawlowski, M. S., Kroupa, P., \& Jerjen, H. 2013, MNRAS, 435, 1928

Pawlowski, M. S., Famaey, B., Merritt, D., \& Kroupa, P. 2015, ApJ, 815, 19

Peng, C. Y., Ho, L. C., Impey, C. D., \& Rix, H.-W. 2002, AJ, 124, 266
Renaud, F., Famaey, B., \& Kroupa, P. 2016, MNRAS, 463, 3637

Sachdeva, S. 2013. MNRAS, 435, 1186

Simon, J. D., \& Geha, M. 2007, ApJ, 670, 313

Taylor, C., Boylan-Kolchin, M., Torrey, P., Vogelsberger, M., \& Hernquist, L. 2016, MNRAS, 461, 3483

Tully, R. B., Libeskind, N. I., Karachentsev, I. D., et al. 2015, ApJ, 802, L25 Weinzirl, T., Jogee, S., Khochfar, S., Burkert, A., \& Kormendy, J. 2009, ApJ, 696, 411

Wouterloot, J. G. A., \& Walmsley, C. M. 1986, A\&A 168, 237

Yang, Y., Hammer, F., Fouquet, S., et al. 2014, MNRAS, 442, 2419 


\section{Appendix A}

This appendix provides information on potentially disturbing sources when collecting data on dwarf galaxy distributions around major hosts. Adopted distances refer to the average values taken from NED, which are displayed for our sources in Col. 7 of Table 1 and Col. 8 of Table 2. Angular offsets between galaxies are converted into projected distances using the distances $(D)$ to the edge-on galaxies of our sample. "Empty field" indicates a large area free of similarly sized galaxies, extending over at least several $100 \mathrm{kpc}$.

$N G C$ 7814: about 1.5 to the east, corresponding to a projected distance of $\approx 420 \mathrm{kpc}$, lies an optically much less prominent galaxy, NGC 14 (distance: $9 \pm 3 \mathrm{Mpc}$ ).

NGC 100: empty field.

UGC 1281: $\approx 5^{\circ}$ off M33; NGC 736 and NGC 750 at offsets of $\approx 2^{\circ}$, corresponding to $\approx 200 \mathrm{kpc}$, in the northwest. Lots of small background galaxies clustering most densely $\approx 15^{\prime}$ to the east and $\approx 30^{\prime}$ to the north.

ESO 0477-G016: NGC 723 (no specific distance estimate, $V=1485 \mathrm{~km} \mathrm{~s}^{-1}$ ) is located $\approx 1^{\circ}$ in the southwest. This angular distance corresponds to $\approx 600 \mathrm{kpc}$. Otherwise empty field.

$N G C$ 891: two relative to NGC 891 quite inconspicious galaxies, NGC $898 \approx 25^{\prime}$ to the south and UGC $1841 \approx 40^{\prime}$ to the north, are with $V \approx 5500$ and $\approx 6375 \mathrm{~km} \mathrm{~s}^{-1}$ far in the background. NGC 906 and NGC 914, $\approx 30^{\prime}$ in the southeast and being similarly inconspicious, have radial velocities of 4680 and $5535 \mathrm{~km} \mathrm{~s}^{-1}$.

NGC 1886: no similarly sized galaxy within $2^{\circ}(1 \mathrm{Mpc})$.

ESO 121-G006: the less conspicious galaxy NGC 2205, $\approx 50^{\prime}$ to the southeast, is with $V=8385 \mathrm{~km} \mathrm{~s}^{-1}$ far in the background. Otherwise empty field.

NGC 2549: empty field.

NGC 2654: there are similarly prominent (w.r.t. angular size) galaxies $2^{\circ}-3^{\circ}$ south (NGC 2685) and east (NGC 2742), corresponding to projected distances of 1.0 and $1.5 \mathrm{Mpc}$.

UGC 5245: an optically slightly more prominent galaxy, NGC2974, is located at similar distance $\approx 2^{\circ}(\approx 700 \mathrm{kpc})$ in the southwest.

\section{NGC 3098: empty field.}

NGC 3115: there are two obvious dwarf companions, PGC 29299 and PGC 29300, $5^{\prime}$ and $15^{\prime}-20^{\prime}$ in the east and southeast, respectively. With respect to galaxies with similar angular extent, empty field.

UGC 5459: the less conspicuous galaxy UGC 5460 is located $\approx 75^{\prime}$ (projected $\approx 500 \mathrm{kpc}$ ) to the south.

NGC 3245A: the companion galaxy NGC 3245 is located at an offset of $\approx 9^{\prime}$ to the south, corresponding to a projected distance of $\approx 65 \mathrm{kpc}$. NGC $3254(D=33 \pm 5 \mathrm{Mpc})$ is $\approx 1$ degree in the north.

NGC 3669: appears to be slightly warped, with the central region edge-on but with slightly less inclined outskirts. $40^{\prime}-50^{\prime}$ to the south and southeast are NGC 3674 and NGC 3683A, 40'$50^{\prime}$ to the west we find NGC 3613 and NGC 3619 and a few smaller galaxies at $30^{\prime}$ in the west. These galaxies may be located at a distance of $\approx 30 \mathrm{Mpc}$, which leads to projected distances $\gtrsim 250 \mathrm{kpc}$.
UGC 6792: LSB galaxy PGC37050 $\approx 1^{\circ}$ (projected $350 \mathrm{kpc}$ ) to the south.

NGC 4179: NGC 4116 and NGC 4123 at similar distance as NGC 4179 (see Table 1 ) are off $\approx 2^{\circ}$ (projected $\approx 600 \mathrm{kpc}$ ) to the northwest. The background source NGC 4073 is also seen at an offset of 2 degrees, but in the west.

$U G C$ 7321: NGC 4204 lies at an offset of $\approx 2^{\circ}(\approx 600 \mathrm{kpc})$ the south. NGC 4455 is seen at an even larger angular distance. However, both galaxies may be closer to us than UGC 7321.

$N G C$ 4244: the less bright galaxy NGC 4214 is located at similar distance $(3 \pm 1 \mathrm{Mpc}) \approx 1.5$ to the south. At the distance to NGC 4244 this corresponds to a projected linear offset of $105 \mathrm{kpc}$. NGC 4145 and NGC 4151 at distances of 15-20 Mpc are located $\approx 2^{\circ}$ in the northwest.

UGC 7394: UGC 7370 with a velocity of $V \approx 2215 \mathrm{~km} \mathrm{~s}^{-1}$ is located $\approx 40^{\prime}(\approx 400 \mathrm{kpc})$ in the northwest. UGC 7396 with a velocity of $2100 \mathrm{~km} \mathrm{~s}^{-1}$ is $\approx 40^{\prime}$ to the south. Both galaxies may be seen at a similar distance as UGC 7394.

$N G C$ 4565: the smaller galaxy NGC 4494 (at similar distance) is located 1.15 to the west, corresponding to a projected offset of $230 \mathrm{kpc}$. NGC 4559 in the north and NGC 4725 in the east, also at similar distances, show angular offsets twice and three times as large.

$N G C$ 4634: a pair with NGC 4633, displaced by less than $4^{\prime}$ to the north. The pair is surrounded by Virgo Cluster galaxies $(D \approx 15 \mathrm{Mpc})$ at angular offsets $\gtrsim 70^{\prime}(\gtrsim 400 \mathrm{kpc})$, that is by M90, M91, NGC 4571, NGC 4639, NGC 4654, NGC 4659, NGC 4689, and NGC 4710.

ESO 575-G061: there are quite a number of (slightly) less bright galaxies like ESO 575-G060 in the field. These have radial velocities in excess of $10000 \mathrm{~km} \mathrm{~s}^{-1}$.

NGC 5170: NGC 5247, likely at similar distance, lies almost $2^{\circ}(\approx 925 \mathrm{kpc})$ in the east.

$U G C$ 8880: next slightly less prominent galaxies are located about $45^{\prime}$ northeast and $55^{\prime}$ northwest.

NGC 5470: the less prominent galaxy NGC 5491 is located $\approx 1^{\circ}(\approx 400 \mathrm{kpc})$ to the east. Several galaxies (e.g. NGC 5374 (background), NGC 5382 (uncertain distance), and NGC 5387 (background)) can be found $\approx 2^{\circ}$ to the west.

$U G C$ 9242: the galaxy is surrounded by several similarly prominent galaxies at $\approx 1^{\circ}$ angular distance $(\approx 350 \mathrm{kpc})$, i.e. by NGC 5582, UGC 9203, and UGC 9291, which may be located at similar or larger distances.

$U G C$ 9249: NGC 5665, $\approx 1.5(\approx 500 \mathrm{kpc})$ in the southeast, and NGC 5669 at almost $2^{\circ}$ northeast are located at distances similar to that of UGC 9249 (see Table 1).

NGC 5746: possibly accompanied by the much smaller galaxy NGC $5740, \approx 18^{\prime}$ to the southwest, corresponding to a projected distance of $160 \mathrm{kpc}$. Other galaxies (NGC 5690, NGC 5719, NGC 5774, and NGC 5775) show offsets of at least $2^{\circ}(\gtrsim 1 \mathrm{Mpc})$ and are located not closer than $\approx 20 \mathrm{Mpc}$.

NGC 5866 and NGC 5907: separated by $\approx 1: 4$ (350 pc). The less conspicious galaxies NGC 5905 and NGC 5908, almost a full degree to the south of NGC 5907 and $\approx 1.4$ east of NGC 5866, belong with $V \approx 3350 \mathrm{~km} \mathrm{~s}^{-1}$ to the background. NGC 5879 may be part of a group composed of NGC 5866 


\section{Henkel et al.: An edge-on sample of nearby galaxies}

and NGC 5907, but is less bright (location: $1^{\circ}$ northwest of NGC 5907 and 1.5 north of NGC 5866).

UGC 9977: UGC $9979,15^{\prime}$ to the south $(\approx 125 \mathrm{kpc})$, may be located at similar distance but is clearly less prominent. Otherwise empty field.
ESO 146-G014: empty field. IC 5176: empty field.

$N G C$ 7332: pair with NGC 7339 in the east (at 5', corresponding to $\approx 20 \mathrm{kpc}$ ), another galaxy viewed close to edge-on. Otherwise empty field. 